\title{
The Peak Algebra of the Symmetric Group
}

\author{
KATHRYN L. NYMAN
}

Received March 5, 2002; Revised October 8, 2002

\begin{abstract}
The peak set of a permutation $\sigma$ is the set $\{i: \sigma(i-1)<\sigma(i)>\sigma(i+1)\}$. The group algebra of the symmetric group $S_{n}$ admits a subalgebra in which elements are sums of permutations with a common descent set. In this paper we show the existence of a subalgebra of this descent algebra in which elements are sums of permutations sharing a common peak set. To prove the existence of this peak algebra we use the theory of enriched $(P, \gamma)$-partitions and the algebra of quasisymmetric peak functions studied by Stembridge (Trans. Amer. Math. Soc. 349 (1997) 763-788).
\end{abstract}

Keywords: peaks, Solomon's descent algebra, quasisymmetric functions

\section{Introduction}

In 1976 Louis Solomon [12] introduced a collection of algebras associated to finite Coxeter groups. In the case of the symmetric group the elements of the associated algebra are sums (in the group algebra of $S_{n}$ over a field $\mathbf{k}$ ) of permutations sharing a common descent set. We refer to this algebra as the descent algebra and denote it by $\operatorname{Sol}\left(A_{n-1}\right)$ (see [3, 8], and [4]). In addition commutative subalgebras of the descent algebra have been studied in which permutations are grouped according to the number of descents ([1, 8, 10], and [6]) and according to the shapes of the permutations [7].

As an analogue to the descent algebra of the symmetric group we look at a subalgebra of the group algebra of $S_{n}$ indexed by the position of peaks in the permutations. To prove the existence of this subalgebra we will utilize a set of maps on labeled posets, introduced by Stembridge [15], called enriched $(P, \gamma)$-partitions which are a variation of Stanley's notion of (ordinary) $P$-partitions [14]. A more in-depth discussion of enriched $(P, \gamma)$-partitions can be found in [13].

We begin with several definitions and notation. We will write permutations of $[n]$ in the form $\sigma=(\sigma(1) \sigma(2) \cdots \sigma(n))$, and for $\sigma, \gamma \in S_{n}$ the product $\sigma \gamma$ will indicate first applying $\gamma$ and then applying $\sigma$. The descent set of a permutation, denoted $D(\sigma)$, is the set $\{i: \sigma(i)>\sigma(i+1)\}$. Thus $D(\sigma) \subseteq[n-1]$. For example the descent set of $\sigma=(31524)$ is $\{1,3\}$. Let $\mathcal{D}_{T}=\sum_{\sigma: D(\sigma)=T} \sigma$. Since there are $2^{n-1}$ possible descent sets, the symmetric group $S_{n}$ is partitioned into $2^{n-1}$ disjoint descent classes. Solomon's result shows the product of two descent classes is a linear combination, with non-negative integer coefficients, of descent classes:

$$
\mathcal{D}_{T} \cdot \mathcal{D}_{Q}=\sum_{K} a_{K} \mathcal{D}_{K} \quad a_{K} \in \mathbb{Z}_{+}
$$


A formal power series $f$ of bounded degree in a countable number of indeterminates $x_{1}, x_{2}, \ldots$ with coefficients in a field $\mathbf{k}$ is a quasisymmetric function if for any $a_{1}, \ldots, a_{k} \in$ $\mathbb{Z}_{+}$, the coefficient of $x_{i_{1}}^{a_{1}} \cdots x_{i_{k}}^{a_{k}}$ in $f$ is equal to the coefficient of $x_{j_{1}}^{a_{1}} \cdots x_{j_{k}}^{a_{k}}$ whenever $i_{1}<\cdots<i_{k}$ and $j_{1}<\cdots<j_{k}$. Clearly symmetric functions are quasisymmetric functions. The series $\sum_{i<j} x_{i} x_{j}^{2}$ and $\sum_{i<j<k} x_{i}^{3} x_{j} x_{k}^{2}$ are examples of quasisymmetric functions that are not symmetric.

Let $Q S y m=\bigoplus_{n>0} \mathcal{Q}_{n}$ denote the algebra of quasisymmetric functions over a field $\mathbf{k}$. For $\alpha=\left(\alpha_{1}, \ldots, \alpha_{k}\right)$ a composition of $n($ denoted $\alpha=n)$ and

$$
M_{\alpha}=\sum_{i_{1}<\cdots<i_{k}} x_{i_{1}}^{\alpha_{1}} \cdots x_{i_{k}}^{\alpha_{k}}
$$

the set $\left\{M_{\alpha}: \alpha \models n\right\}$ forms a basis for $\mathcal{Q}_{n}$. Since there is a bijection between compositions of $n$ and subsets of $[n-1]$, we will sometimes write $M_{E}$ for $E \subseteq[n-1]$ to denote the basis element $M_{\alpha}$ where $\alpha$ is the composition of $n$ corresponding to $E$. We will also use Gessel's fundamental basis [9],

$$
F_{D}=\sum_{D \subseteq E} M_{E}
$$

For $f \in \mathcal{Q}_{m}$ and $g \in \mathcal{Q}_{n}$, the product $f g \in \mathcal{Q}_{m+n}$ making $Q S y m$ a graded k-algebra.

\section{The peak algebra of the symmetric group}

We say that a permutation $\gamma \in S_{n}$ has a peak at position $i$ if $\gamma(i-1)<\gamma(i)>\gamma(i+1)$. The peak set of $\gamma$ is the set

$$
\Lambda(\gamma):=\{i: \gamma(i-1)<\gamma(i)>\gamma(i+1)\}
$$

For example the permutation $\gamma=(325461) \in S_{6}$ has peaks at positions 3 and 5 since $\gamma(2)<\gamma(3)>\gamma(4)$ and $\gamma(4)<\gamma(5)>\gamma(6)$. We note that every subset $S$ of $[n-1]$ is the descent set of at least one permutation of $[n]$, and every subset $\Gamma$ of $[n-1]$ satisfying $1 \notin \Gamma$ and $k \in \Gamma$ implies $(k+1) \notin \Gamma$ is the peak set of at least one permutation of $[n]$. For this reason we refer to arbitrary subsets of $[n-1]$ as descent sets and to subsets $\Gamma$ of $[n-1]$ with $1 \notin \Gamma$ and $k \in \Gamma$ implies $(k+1) \notin \Gamma$ as peak sets. We remark here that it is possible to allow peaks to occur at position 1 and this forms the basis of future work.

Given a descent set $S=\left\{s_{1}, \ldots, s_{k}\right\} \subseteq[n-1]$ we form the corresponding peak set $\Lambda^{\prime}(S)$ by removing each $s_{i}$ such that $s_{i}-s_{i-1}=1$, where $s_{0}=0$. For example the descent set $S=\{1,3,4,7,8\}$ gives rise to the peak set $\Lambda^{\prime}(S)=\{3,7\}$.

Our main result shows the existence of a subalgebra of the descent algebra of $S_{n}$ in which elements are sums of permutations sharing a peak class.

Theorem 1 In the group algebra $\mathbb{Q} S_{n}$ of $S_{n}$, define for each peak set $\Gamma \subset[n-1]$

$$
P_{\Gamma}=\sum_{w: \Lambda(w)=\Gamma} w
$$


where $\Lambda(w)$ is the peak set of $w \in S_{n}$. Then the subspace $\mathcal{P}_{n}$ spanned by the $P_{\Gamma}$ 's is a subalgebra of Sol $\left(A_{n-1}\right)$. We call this the peak algebra.

Example 2 Consider the peak algebra of $S_{4}$. $\mathcal{P}_{4}$ consists of 3 elements: permutations with no peaks, permutations with a peak at position 2 and permutations with a peak at position 3 . We will denote these three classes by $P_{\emptyset}, P_{2}$ and $P_{3}$ respectively.

$$
\begin{aligned}
P_{\emptyset}= & (1234)+(2134)+(3124)+(4123)+(4312)+(3214)+(4213)+(4321) \\
P_{2}= & (1432)+(2431)+(3421)+(1423)+(2413)+(3412)+(1324)+(2314) \\
P_{3}= & (1243)+(1342)+(2341)+(4132)+(4231)+(3241)+(3142)+(2143) \\
P_{3} \cdot P_{2}= & (1342)+(1243)+(2143)+(4231)+(4132)+(3142)+(3241) \\
& +(2341)+(2341)+(3241)+(3142)+(1234)+(2134)+(2143) \\
& +(1243)+(1342)+(4321)+(4231)+(4132)+(3214)+(3124) \\
& +(4123)+(4213)+(4312)+(1324)+(1234)+(2134)+(4213) \\
& +(4123)+(3124)+(3214)+(2314)+(2314)+(3214)+(3124) \\
& +(1243)+(2143)+(2134)+(1234)+(1324)+(4312)+(4213) \\
& +(4123)+(3241)+(3142)+(4132)+(4231)+(4321)+(1423) \\
& +(1432)+(2431)+(4312)+(4321)+(3421)+(3412)+(2413) \\
& +(2413)+(3412)+(3421)+(1342)+(2341)+(2431)+(1432) \\
& +(1423) \\
= & 3 P_{\emptyset}+2 P_{2}+3 P_{3}
\end{aligned}
$$

The multiplication in $\mathcal{P}_{4}$ is summarized in Table 1 in which $(x, y, z)$ refers to the sum $x P_{\emptyset}+y P_{2}+z P_{3}$. We note here that a combinatorial multiplication rule which gives the coefficients $a_{k}$, where $P_{i} \cdot P_{j}=\sum_{k} a_{k} P_{k}$ is still an open question.

Denote by $(P, \gamma)$ the labeled poset $\mathrm{P}$ with labels $\gamma(x)$ for $x \in P$, where $\gamma$ is an injective map to a set of totally ordered elements (we will take this set to be $[n]$ where $n=|P|$; see figure 1). We represent $x<y$ in a poset if there is a path between $x$ and $y$ and if $x$ is "under" $y$.

For a poset $P$ with $n$ elements, a linear extension $\bar{v}=\left\{v_{1}<\cdots<v_{n}\right\}$ is a total ordering of the elements of $P$ that preserves the partial order of $P$, i.e., $v_{k}<v_{j}$ in $P$ implies $v_{k}<v_{j}$ in $\bar{v}$. We next consider the set of linear extensions of a labeled poset $(P, \gamma)$ regarded as permutations of the labels of $P$.

Table 1. The multiplication of $\mathcal{P}_{4}$.

\begin{tabular}{cccc}
\hline$\cdot$ & $P_{\emptyset}$ & $P_{2}$ & $P_{3}$ \\
\hline$P_{\emptyset}$ & $(4,2,2)$ & $(2,3,3)$ & $(2,3,3)$ \\
$P_{2}$ & $(2,4,2)$ & $(3,2,3)$ & $(3,2,3)$ \\
$P_{3}$ & $(2,2,4)$ & $(3,3,2)$ & $(3,3,2)$ \\
\hline
\end{tabular}




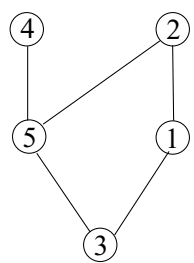

Figure 1. A labeled poset $(P, \gamma)$

Definition 3 Recall for a labeled poset $(P, \gamma)$ the Jordan-Hölder set $\mathcal{L}(P, \gamma)$ is

$$
\mathcal{L}(P, \gamma)=\left\{\left(\gamma\left(v_{1}\right) \ldots \gamma\left(v_{n}\right)\right): v_{1}<\cdots<v_{n} \text { is a linear extension of } P\right\} .
$$

For example the Jordan-Hölder set of the labeled poset $(P, \gamma)$ in figure 1 is $\mathcal{L}(P, \gamma)=$ $\{(35142),(35124),(35412),(31524),(31542)\}$.

Now let $\mathbb{P}$ denote the set of non-zero integers with the total ordering:

$$
-1 \prec 1 \prec-2 \prec 2 \prec-3 \prec 3 \prec \ldots
$$

We will say $k>0$ to indicate positive integers and $|k|$ to refer to the absolute value of an integer as usual.

Definition 4 (Stembridge [15]) For $(P, \gamma)$ a labeled poset, an enriched $(P, \gamma)$-partition is a map $f: P \longrightarrow \mathbb{P}$ such that for all $x<y$ in $P$, we have:

1. $f(x) \preceq f(y)$,

2. $f(x)=f(y)>0$ implies $\gamma(x)<\gamma(y)$,

3. $f(x)=f(y)<0$ implies $\gamma(x)>\gamma(y)$.

Let $\mathcal{E}(P, \gamma)$ denote the set of enriched $(P, \gamma)$-partitions. Figure 2 gives an example of an enriched $(P, \gamma)$-partition in which the $\gamma$ labels appear in the vertices while $f(x)$ is shown below each element $x$.

We can think of $v \in \mathcal{L}(P, \gamma)$ as a labeled poset by considering $\gamma$ as labels on the underlying chain formed by the linear extension $v_{1}<\cdots<v_{n}$. In this way we can define enriched $(v, \gamma)$-partitions.

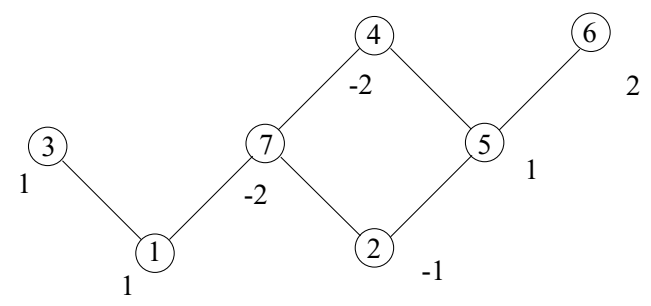

Figure 2. An enriched $(P, \gamma)$-partition $f$. 
Lemma 5 (Stembridge [15]) For any labeled poset $(P, \gamma)$, we have

$$
\mathcal{E}(P, \gamma)=\bigcup_{v \in \mathcal{L}(P, \gamma)} \mathcal{E}(v, \gamma)
$$

where $\mathcal{E}(v, \gamma)$ is the set of enriched $(v, \gamma)$-partitions for the labeled chain $v$.

For each enriched $(P, \gamma)$-partition Stembridge [15] defines a weight enumerator that assigns the weight $z_{k}$ to both $k$ and $-k$. Taking the product of weights for $f(x)$ over all $x \in P$, summed over all $(P, \gamma)$-partitions gives a homogeneous quasisymmetric function:

$$
\Delta(P, \gamma):=\sum_{f \in \mathcal{E}(P, \gamma)} \prod_{x \in P} z_{|f(x)|} .
$$

By Lemma 5 we have

$$
\Delta(P, \gamma)=\sum_{v \in \mathcal{L}(P, \gamma)} \Delta(v, \gamma) .
$$

Stembridge demonstrates that $\Delta(v, \gamma)$ depends only on the peak set of $v \in \mathcal{L}(P, \gamma)[15$, Proposition 2.2], that is, the set

$$
\Lambda(v):=\left\{i: \gamma\left(v_{i-1}\right)<\gamma\left(v_{i}\right)>\gamma\left(v_{i+1}\right), 1<i<n\right\} .
$$

Following Stembridge define a family of quasisymmetric functions

$$
\Theta_{\Lambda}:=\Delta(v, \gamma)
$$

for any labeled chain $(v, \gamma)$ such that $\Lambda(v)=\Lambda$. These $\Theta_{\Lambda}$ s form a basis of the algebra of quasisymmetric peak functions studied by Stembridge and discussed in Section 3 [15]. In this case we can rewrite (2) as

$$
\Delta(P, \gamma)=\sum_{v \in \mathcal{L}(P, \gamma)} \Theta_{\Lambda(v)} .
$$

Notice in calculating the weight enumerator $\Delta$, only the absolute values of the mapping $f: P \rightarrow \mathbb{P}$ are of concern. As in [15] we will call two labelings $\gamma$ and $\gamma^{\prime}$ of a poset $P$ weakly equivalent if $\{|f|: f \in \mathcal{E}(P, \gamma)\}=\left\{|f|: f \in \mathcal{E}\left(P, \gamma^{\prime}\right)\right\}$ as multisets, where $|f|(x):=|f(x)|$. Thus $\Delta(P, \gamma)=\Delta\left(P, \gamma^{\prime}\right)$ for weakly equivalent labelings $\gamma$ and $\gamma^{\prime}$.

A criterion of Stembridge [15, Proposition 2.4] for testing weak equivalence of labelings involves the order ideals of a poset $P$, that is the subsets $I \subseteq P$ such that if $x \in I$ and $y<x$ then $y \in I$. For a labeled poset $(P, \gamma)$, Stembridge defines binary relations $\rightarrow$ and $\leftarrow$ on the set, $J(P)$, of order ideals of $P$ by

$$
\begin{array}{ll}
I \rightarrow J & \text { if } I \subset J, \text { and } x, y \in J \backslash I, x<y \Rightarrow \gamma(x)>\gamma(y), \\
I \leftarrow J & \text { if } I \subset J, \text { and } x, y \in J \backslash I, x<y \Rightarrow \gamma(x)<\gamma(y) .
\end{array}
$$


And finally, for any $I \subset J$,

$$
N(P, \gamma)(I, J):=|\{K \in J(P): I \rightarrow K \leftarrow J\}| .
$$

A subposet $Q$ of $P$ is convex if for $x<y<z$ in $P$ and $x, z \in Q$ we have $y \in Q$ [15].

Proposition 6 (Stembridge) If $\gamma$ and $\gamma^{\prime}$ are labelings of a poset $P$, the following are equivalent:

1. $\gamma$ and $\gamma^{\prime}$ are weakly equivalent.

2. $\Delta(Q, \gamma)=\Delta\left(Q, \gamma^{\prime}\right)$ for every convex subposet $Q$ of $P$.

3. $N(P, \gamma)=N\left(P, \gamma^{\prime}\right)$.

We will omit the $(P, \gamma)$ in the notation of $N(P, \gamma)$ when there is only one poset and labeling under consideration.

Definition 7 A range poset is a poset whose Hasse diagram is a path. As such each element belongs to $\leq 2$ maximal chains with equality possible only for maximal or minimal elements of $P$.

A range poset has a natural left to right ordering on its elements as well as the usual partial ordering. Given a descent set $S \subseteq[n-1]$ we define the range poset $M_{S}$ corresponding to $S$ to be the poset such that if the elements are labeled $1, \ldots, n$ from left to right, then element $i>i+1$ if and only if $i \in S$. Figure 3 illustrates the range poset corresponding to the descent set $\{1,4,6,7\}$. Given a permutation $\gamma \in S_{n}$ there is a natural labeling of an $n$ element range poset $P$ by assigning $\gamma(i)$ to the $i$ th element from the left end of $P$.

Remark 8 For the remainder of this paper a peak of $\gamma$ will refer to a peak of a permutation $\gamma$ (i.e., $3<5>4$ ) and a trough of $\gamma$ will refer to a trough of the permutation (i.e., $2>1<6$ ). A hill (respectively, a valley) of a range poset $P$ will represent a maximal (minimal) element of $P$.

The next lemma can be found in [13, Exercise 7.95a]. The proof of Theorem 1 is an extension of the solution to [13, Exercise $7.95 \mathrm{~b}]$ in which Gessel uses ordinary $P$-partitions to prove the existence of Solomon's descent algebra. When extending to enriched $(P, \gamma)-$ partitions it is necessary to work with the union of range posets which share a common hill

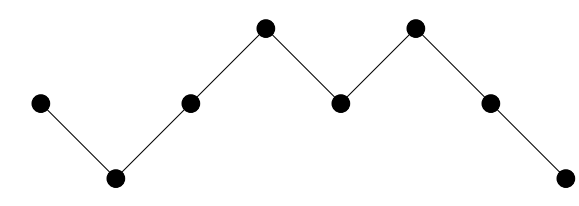

Figure 3. A range poset. 


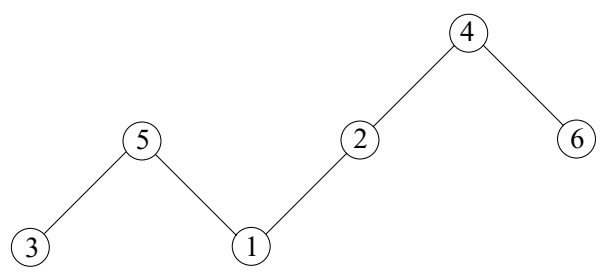

Figure 4. A range poset $M_{\{2,5\}}$ labeled by $\gamma=(351246)$.

set. In addition, to show the dependence of the weight enumerator of the enriched $(P, \gamma)$ partitions on the peak set of the labeling permutation we will require a result on the weak equivalence of labelings of range posets. We will give this result in Corollary 12.

Lemma 9 (Stanley) Given $S \subseteq[n-1]$ and $\gamma \in S_{n}$, let $\left(M_{S}, \gamma\right)$ be the labeled range poset corresponding to $S$. Then the Jordan-Hölder set $\mathcal{L}\left(M_{S}, \gamma\right)$ consists of all permutations $v \in S_{n}$ such that $D\left(v^{-1} \gamma\right)=S$.

To illustrate this lemma consider the labeled range poset $\left(M_{\{2,5\}}, \gamma\right)$ in figure 4 where $\gamma=(351246)$. Let $v=(135264) \in \mathcal{L}\left(M_{\{2,5\}}, \gamma\right)$. Then $v^{-1} \gamma=(231465)$, and $D\left(v^{-1} \gamma\right)=$ $\{2,5\}$. We can see that this result is true by calling the $i$ th element from the left end of $M_{S}$ element $i$. Then $v^{-1} \gamma(i)$ gives the position of $\gamma(i)$ in $v$. Therefore a descent of $v^{-1} \gamma$ at position $j$ indicates $\gamma(j+1)$ appears before $\gamma(j)$ in $v$ and hence $j>(j+1)$ in $M_{S}$.

A similar result holds if we replace descent sets with peak sets. Here we must consider the set of all range posets with a particular peak set. For a peak set $\Gamma \subset[n-1]$ let $\mathcal{S}_{\Gamma}=\left\{S \subseteq[n-1]: \Lambda^{\prime}(S)=\Gamma\right\}$ be the collection of descent sets having peak set $\Gamma$. And let $\mathcal{M}_{\Gamma}=\left\{M_{S}: S \in \mathcal{S}_{\Gamma}\right\}$ be the family of range posets with descents at $\left\{S: S \in \mathcal{S}_{\Gamma}\right\}$.

Corollary 10 Given a peak set $\Gamma \subset[n-1]$ and a fixed permutation $\gamma \in S_{n}$ consider the family of labeled posets $\left\{\left(M_{S}, \gamma\right): M_{S} \in \mathcal{M}_{\Gamma}\right\}$. The union of Jordan-Hölder sets $\bigcup_{\mathcal{M}_{\Gamma}} \mathcal{L}\left(M_{S}, \gamma\right)$ consists of all permutations $v \in S_{n}$ such that $\Lambda\left(v^{-1} \gamma\right)=\Gamma$.

Proof: By Lemma 9, a permutation $v \in \mathcal{L}\left(M_{S}, \gamma\right)$, for $M_{S} \in \mathcal{M}_{\Gamma}$, if and only if the descent set $D\left(v^{-1} \gamma\right)=S$. Note if $S=D(u)$ then $\Lambda^{\prime}(S)=\Lambda(u)$. Since $\Lambda^{\prime}(S)=\Gamma$ for all $S \in \mathcal{S}_{\Gamma}$, any $v \in \bigcup_{\mathcal{M}_{\Gamma}} \mathcal{L}\left(M_{S}, \gamma\right)$ has $\Lambda\left(v^{-1} \gamma\right)=\Gamma$. Conversely, a permutation $v$ such that $\Lambda\left(v^{-1} \gamma\right)=\Gamma$ has the property that $D\left(v^{-1} \gamma\right)=S$ for some $S \in \mathcal{S}_{\Gamma}$. Hence $v \in \mathcal{L}\left(M_{S}, \gamma\right)$ and thus is in $\bigcup_{\mathcal{M}_{\Gamma}} \mathcal{L}\left(M_{S}, \gamma\right)$.

Next we give a formula for $N(P, \gamma)(I, J)$, where $P$ is a range poset, in terms of the positions of peaks of the labeling permutation $\gamma$. Notice that for a range poset $P$ order ideals are unions of disjoint components, where a component consists of a set of consecutive elements of $P$ (reading elements from left to right). These components are separated by hills of $P$. Similarly, for two order ideals $I \subseteq J$ the set $J \backslash I$ is composed of disjoint components separated by either hills or valleys of $P$ (see figure 5). 


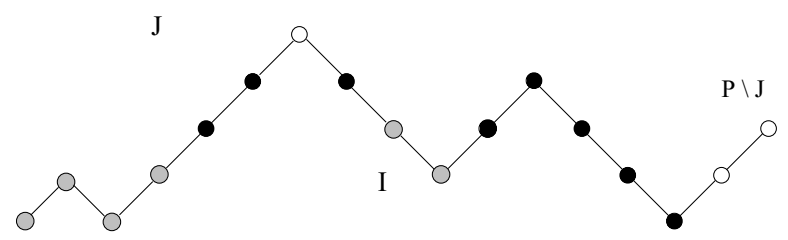

Figure 5. Order ideals $I \subset J$ of a range poset $P$. Ideal $I$ is shaded, $J \backslash I$ is in black, and $P \backslash J$ is white.

We can think of a component $M$ as a subposet of $P$. The interior of $M$ will refer to elements which are not the first or last elements of $M$ (reading from left to right). Interior hills or valleys of $M$ will thus be maximal or minimal elements of $P$ which are interior elements of $M$, and an interior slope of $M$ is composed of interior elements which are neither maximal nor minimal elements of $P$. Given a component $M$, interior peaks or troughs of a labeling $\gamma$ will refer to peaks or troughs of $\gamma$ which occur on interior elements of $M$.

Proposition 11 For $P$ a range poset labeled by $\gamma \in S_{n}$ and $I \subset J$ order ideals

$$
N(P, \gamma)(I, J)=\prod_{M} k_{M}
$$

where the product is taken over the components $M$ of $J \backslash I$ and

$$
k_{M}= \begin{cases}0 & \text { if there is a peak of } \gamma \text { on an interior slope of } M \\ 2^{\vartheta+1} & \text { otherwise }\end{cases}
$$

for $\vartheta$ the number of peaks of $\gamma$ on interior hills and valleys of $M$.

Corollary 12 For a range poset $M_{S}$, two labelings $\gamma, \gamma^{\prime} \in S_{n}$ are weakly equivalent if and only if $\Lambda(\gamma)=\Lambda\left(\gamma^{\prime}\right)$.

Proof: For a range poset $M_{S}$, and arbitrary order ideals $I \subseteq J$, Proposition 11 gives $N\left(M_{S}, \gamma\right)(I, J)$ in terms of the positions of peaks of $\gamma$. Thus if $\Lambda(\gamma)=\Lambda\left(\gamma^{\prime}\right)$ we have $N\left(M_{S}, \gamma\right)=N\left(M_{S}, \gamma^{\prime}\right)$. So by Proposition $6, \gamma$ and $\gamma^{\prime}$ are weakly equivalent.

For the converse we show that if $\Lambda(\gamma) \neq \Lambda\left(\gamma^{\prime}\right)$ then $N\left(M_{S}, \gamma\right) \neq N\left(M_{S}, \gamma^{\prime}\right)$. Suppose $i \in \Lambda(\gamma), i \notin \Lambda\left(\gamma^{\prime}\right)$. Take order ideals $I \subset J$ such that $J \backslash I$ consists of the elements $i-1$, $i, i+1$ of $M_{S}$ (reading left to right). Since $i \notin \Lambda\left(\gamma^{\prime}\right), \gamma^{\prime}$ has no peaks in the interior of $J \backslash I$. By Proposition 11, if element $i$ is on a slope of $M_{S}$ (and hence an interior slope of $J \backslash I), N\left(M_{S}, \gamma\right)(I, J)=0$ while $N\left(M_{S}, \gamma^{\prime}\right)(I, J)=2$. If $i$ is on a hill or valley of $M_{S}$ (and hence an interior hill or valley of $J \backslash I), N\left(M_{S}, \gamma\right)(I, J)=2^{2}$ while $N\left(M_{S}, \gamma^{\prime}\right)(I, J)=2$. Thus $N\left(M_{S}, \gamma\right) \neq N\left(M_{S}, \gamma^{\prime}\right)$.

We are now in a position to prove Theorem 1 and Proposition 11. 
Proof of Theorem 1: We show that the product of two peak classes is again a sum of peak classes; that is

$$
P_{\Upsilon} \cdot P_{\Gamma}=\sum_{i} P_{\Theta_{i}} \cdot
$$

If $\gamma$ and $\gamma^{\prime}$ have the same peak set they must appear the same number of times on the right of (7), so we must show for $\gamma, \gamma^{\prime} \in S_{n}$ with $\Lambda(\gamma)=\Lambda\left(\gamma^{\prime}\right)$

$$
\begin{aligned}
& \left|\left\{(u, v) \in S_{n} \times S_{n}: v u=\gamma, \Lambda(u)=\Gamma, \Lambda(v)=\Upsilon\right\}\right| \\
& \quad=\left|\left\{(u, v) \in S_{n} \times S_{n}: v u=\gamma^{\prime}, \Lambda(u)=\Gamma, \Lambda(v)=\Upsilon\right\}\right| .
\end{aligned}
$$

And so, for each $\gamma$, the number of such pairs $(u, v)$ in $S_{n} \times S_{n}$ as above, should depend only on the peak sets $\Gamma, \Upsilon$, and $\Lambda(\gamma)$.

For $\gamma \in S_{n}$ and $S \subseteq[n-1]$ with $\Lambda^{\prime}(S)=\Gamma$, consider the set $\mathcal{E}\left(M_{S}, \gamma\right)$ of enriched $\left(M_{S}, \gamma\right)$-partitions. By (4) the weight enumerator $\Delta\left(M_{S}, \gamma\right)=\sum_{v \in \mathcal{L}\left(M_{s}, \gamma\right)} \Theta_{\Lambda(v)}$ is a sum of basis elements of the algebra of quasisymmetric peak functions, and this representation is unique. Thus the multiset $\left\{\Lambda(v): v \in \mathcal{L}\left(M_{S}, \gamma\right)\right\}$ is determined by $\Delta\left(M_{S}, \gamma\right)$.

If we consider the sum of weight enumerators taken over the union of enriched $\left(M_{S}, \gamma\right)$ partitions for all $M_{S} \in \mathcal{M}_{\Gamma}$, we again have a unique representation in terms of the basis elements $\Theta_{\Lambda}$, and so the multiset

$$
\Phi=\bigcup_{M_{S} \in \mathcal{M}_{\Gamma}}\left\{\Lambda(v): v \in \mathcal{L}\left(M_{S}, \gamma\right)\right\}=\left\{\Lambda(v): v \in \bigcup_{M_{S} \in \mathcal{M}_{\Gamma}} \mathcal{L}\left(M_{S}, \gamma\right)\right\}
$$

depends only on

$$
\sum_{M_{S} \in \mathcal{M}_{\Gamma}} \Delta\left(M_{S}, \gamma\right)
$$

This sum in turn depends only upon the weak isomorphism classes of the pairs $\left(M_{S}, \gamma\right)$. By Corollary 12 these weak equivalence classes depend only on the posets $M_{S}$ and the peak set $\Lambda(\gamma)$, while the posets $\left\{M_{S}: M_{S} \in \mathcal{M}_{\Gamma}\right\}$ are completely determined by $\Gamma$.

By Corollary 10 we can conclude that as a multiset,

$$
\Phi=\left\{\Lambda(v): \Lambda\left(v^{-1} \gamma\right)=\Gamma\right\},
$$

which by the above argument depends only upon $\Gamma$ and $\Lambda(\gamma)$. Thus letting $u=v^{-1} \gamma$ we have the number of $u, v$ for which $\Lambda(u)=\Gamma, \Lambda(v)=\Upsilon$, and $v u=\gamma$ depends only on $\Gamma$, $\Upsilon$ and $\Lambda(\gamma)$.

The subspace $\mathcal{P}_{n}$ forms a subalgebra of the descent algebra of $S_{n}$ since elements of $\mathcal{P}_{n}$ are sums of descent classes. Specifically $P_{\Gamma}=\sum_{\Lambda^{\prime}(S)=\Gamma} \mathcal{D}_{S}$ where $\mathcal{D}_{S}=\sum_{D(\gamma)=S} \gamma$.

Proof of Proposition 11: It is sufficient to consider one component of $J \backslash I$ as $N(I, J)$ will be the product of the number of $K$ satisfying $I \rightarrow K \leftarrow J$ in each component. For the remainder of this proof $K$ will refer to an order ideal satisfying $I \rightarrow K \leftarrow J$. 
1)

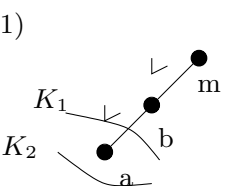

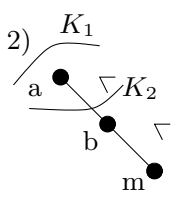

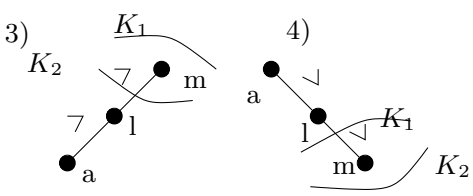

Figure 6. Choices for $K$ in conditions 1 to 4.

Consider an arbitrary segment $M$ of $J \backslash I$. We first note several conditions which any $K$ must satisfy. These conditions are illustrated in figure 6 in which $<$ and $>$ on edges indicate the relative values of $\gamma$.

1. If $\gamma$ is increasing on an increasing slope of $M$, that is if we have $a<b<\cdots<m$ and $\gamma(a)<\gamma(b)<\cdots<\gamma(m)$ then $b$ can not be in $K$. Otherwise we have $a, b \in K \backslash I$ with $a<b$ and $\gamma(a)<\gamma(b)$, violating (5).

2. If $\gamma$ is increasing on an decreasing slope of $M$, that is $a>b>\cdots>m$ and $\gamma(a)<$ $\gamma(b)<\cdots<\gamma(m)$ then $b$ must be in $K$. Otherwise we have $a, b \in J \backslash K$ with $a>b$ and $\gamma(a)<\gamma(b)$, violating (6).

3. Similarly, if $\gamma$ is decreasing on an increasing slope of $M$, namely $a<\cdots<l<m$ and $\gamma(a)>\cdots>\gamma(l)>\gamma(m)$, then $l$ must be in $K$.

4. Finally, if $\gamma$ is decreasing on an decreasing slope of $M$, so $a>\cdots>l>m$ and $\gamma(a)>\cdots>\gamma(l)>\gamma(m)$, then $l$ cannot be in $K$.

First consider the case in which there exists a peak of $\gamma$ on an interior slope of $M$; that is $a<b<c$ in $M$ with $\gamma(a)<\gamma(b)>\gamma(c)$. It cannot be the case that $b \in K$, since $b \in K \backslash I$ implies $a<b \in K \backslash I$ and $\gamma(a)<\gamma(b)$ contrary to (5). But it also cannot be the case that $b \notin K$, since that implies $b<c \in J \backslash K$, with $\gamma(b)>\gamma(c)$ contradicting (6). Thus there is no $K$ satisfying $I \rightarrow K \leftarrow J$, and consequently $N(I, J)=0$.

For the remainder of this proof we assume any peaks of $\gamma$ in the interior of $M$ occur on hills or valleys of $M$. Between each consecutive pair of such peaks there exists a trough of $\gamma$ and we claim regardless of where such a trough is located there are exactly two choices for $K$ satisfying $I \rightarrow K \leftarrow J$. Figure 7 illustrates the choices for $K$ in Cases A, B, and C.

Case A: There exists a trough of $\gamma$ on a slope of $M$. So $a<b<c$ with $\gamma(a)>\gamma(b)<\gamma(c)$.

First note that we cannot have both $a$ and $b$ in $J \backslash K$ since $a<b$ with $\gamma(a)>\gamma(b)$. Thus element $a$ must belong to $K$, but we have a choice to include $b$ or not include $b$ in $K$
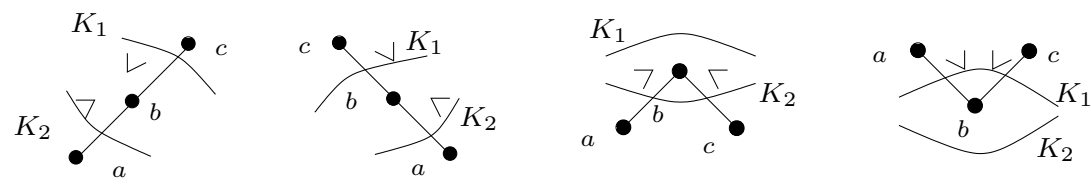

Figure 7. Choices for $K$ in Cases A, B, and C. 
as follows. If $b$ is in $K$ we have $\gamma\left(x_{1}\right)>\gamma\left(x_{2}\right)$ for all $x_{1}<x_{2} \leq b$ in $M$ since there are no peaks of $\gamma$ on internal slopes of $M$. Thus $b \in K$ is consistent with (5). If $b$ is not in $K$, and since $\gamma$ only peaks at hills or valleys of $M$ we have $\gamma\left(y_{1}\right)<\gamma\left(y_{2}\right)$ for all $b \leq y_{1}<y_{2}$ in $M$, which is consistent with (6). We note that $c$ can not belong to $K$ else we have $b<c$ in $K \backslash I$ with $\gamma(b)<\gamma(c)$ a contradiction to (5).

Case B: There exists a trough of $\gamma$ on an interior peak of $M$, i.e., $a<b>c$ with $\gamma(a)>\gamma(b)<\gamma(c)$. Either $a$ or $c \notin K$ violates (6) and again since interior peaks of $\gamma$ only occur at hills or valleys of $M$ we know that $\gamma\left(x_{1}\right)>\gamma\left(x_{2}\right)$ for all $x_{1}<x_{2} \leq b$ in $M$. Thus $b \in K$ or $b \notin K, a, c \in K$ both form valid choices for $K$.

Case $C$ : There exists a trough of $\gamma$ at an interior valley of $M$, i.e., $a>b<c$ with $\gamma(a)>\gamma(b)<\gamma(c)$. We know $\gamma\left(y_{1}\right)>\gamma\left(y_{2}\right)$ for all $y_{1}>y_{2} \geq b$ since interior peaks of $\gamma$ are restricted to hills and valleys of $M$. Thus $b \notin K$ or $b \in K, a, c \notin K$ are valid choices for $K$. Furthermore $a$ or $c \in K$ violate (5).

Hence there are two valid choices for $K$ between each pair of consecutive $\gamma$ peaks. These choices are independent because each choice is separated by either a valley or hill of $M$. We have left to consider the portion of $M$ before the first $\gamma$ peak and the portion of $M$ after the last peak. (If there does not exist an interior $\gamma$ peak on $M$ then $M$ can be treated as one of an initial segment or a final segment.)

If $\gamma$ begins with a descent in the initial segment of $M$ then there exists a trough of $\gamma$ before the first $\gamma$ peak and thus as in Cases A, B, and C there are exactly two choices for $K$. Otherwise $\gamma$ ascends monotonically to the first peak. Then by condition 2 if $M$ contains any decreasing slopes $K$ must include all elements of the slope except (possibly) the top element. And by condition $1, K$ cannot include any element, except (possibly) the smallest, of any increasing slope of $M$. These requirements determine membership in $K$ for all but the initial element $i$ of $M$ (see figure 8). If $M$ begins with an ascent $i<j$ then $i$ may belong to $K$, since $j$ is not in $K$ (by condition 1). But both $i$ and $j$ are eligible as members of $J \backslash K$ so there are two choices for $K$. If $M$ begins with a descent $i>j$ then $i$ may belong to $J \backslash K$ since $j$ must be in $K$ (by condition 2), or both $i$ and $j$ may belong to $K$ since $i>j$ and $\gamma(i)<\gamma(j)$. So again there are 2 possible choices for $K$.

In the final segment of $M, \gamma$ either has a final trough in which case we have two choices for $K$ as before, or $\gamma$ decreases monotonically to the end of $M$. In this situation by reading the elements of $M$ from right to left, and consequently reading $\gamma$ in reverse, this case reduces to the case in which $\gamma$ increases on the initial segment of $M$. And so we have two choices for $K$ by either including or not including the final element (from left to right) of $M$.

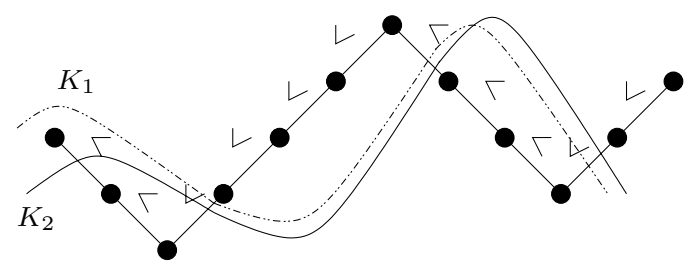

Figure 8. The two possible $K$ s satisfying $I \rightarrow K \leftarrow J$ for $\gamma$ increasing on a segment $M$ of $J \backslash I$. 


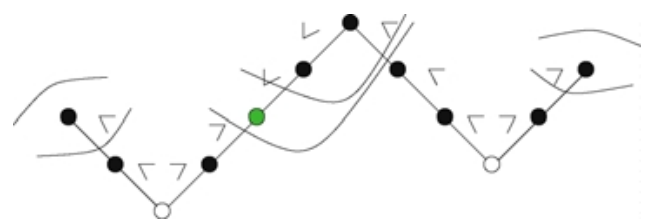

Figure 9. A range poset with $\gamma$ peaks unfilled, and troughs shaded along with choices for where $K$ "splits the trough" and end choices for $K$.

Thus there are two independent choices for $K$ between each pair of consecutive $\gamma$ peaks which occur at interior hills or valleys of $M$ plus two choices for $K$ at both the beginning and end of $M$ for a total of $2^{\vartheta+1}$ such $K$, where $\vartheta$ is the number of peaks of $\gamma$ occurring at interior hills or valleys of $M$ (see figure 9 ).

\section{Connections to Stembridge's peak algebra}

Let $\Pi_{n}$ be the $\mathbb{Z}$-module generated by the $\left\{\Theta_{\Lambda}\right\}$ (3) where $\Lambda$ ranges over peak sets in $[n-1]$, and let $\Pi:=\bigoplus_{n>0} \Pi_{n}$. This defines a graded Hopf subalgebra of $Q S y m$ studied by Stembridge [15] and known as the algebra of peaks. Let $\mathcal{P}=\bigoplus_{n \geq 0} \mathcal{P}_{n}$. Although the peak algebra studied in this paper deals with a multiplication that is defined within each $\mathcal{P}_{n}$ (referred to as the inner multiplication), there are ties to Stembridge's peak algebra which we now address.

We denote by $S y m$ the graded ring of symmetric functions and by $\Omega$ the subring generated by Schur Q-functions (see [13]). Stembridge [15] introduces a map $\theta: Q S y m \rightarrow \Pi$ defined linearly on each graded piece by

$$
\theta\left(F_{D}\right)=\Theta_{\Lambda^{\prime}(D)}
$$

for each $D \subset[n-1]$. Furthermore there is map $\hat{\theta}: S y m \rightarrow \Omega$, and for $\lambda=\left(\lambda_{1}, \lambda_{2}, \ldots\right)$ a partition of $n, \hat{\theta}$ is defined by

$$
\hat{\theta}\left(p_{\lambda}\right)= \begin{cases}2^{|\lambda|} p_{\lambda} & \text { if all parts of } \lambda \text { are odd } \\ 0 & \text { if any part of } \lambda \text { is even }\end{cases}
$$

where $|\lambda|$ is the length of $\lambda, p_{\lambda}=p_{\lambda_{1}} p_{\lambda_{2}}, \ldots$, and $p_{n}$ is the power sum symmetric function, namely $p_{n}=\sum_{i} x_{i}^{n}$. The diagram of figure 10, in which the horizontal maps are inclusions and the vertical maps surjections, commutes [15, Remark 3.2].

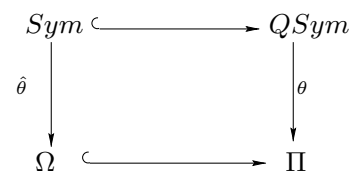

Figure 10. The algebra of peaks and the Schur Q algebra. 


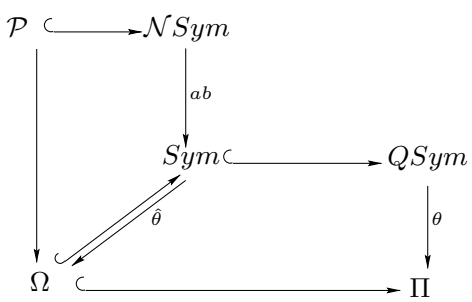

Figure 11. The dualization of figure 10 .

Recall that the Hopf algebra $\mathcal{N} S y m$ is the graded dual of $Q S y m$ via $\mathcal{D}_{K}=F_{K}^{*}$ [11]. And the dual of the inclusion map Sym $\hookrightarrow Q S y m$ is the abelianization map from $\mathcal{N} S y m$ to $S y m$ (recall Sym is a self dual Hopf algebra). Now we identify $\mathcal{P}$ as the dual vector space to $\Pi$ by letting $P_{S}=\Theta_{S}^{*}$. Then as $\mathcal{D}_{K}$ is dual to $F_{K}$, the map $\theta$ is dual to the inclusion map $i\left(P_{S}\right)=\sum_{\Lambda^{\prime}(J)=S} \mathcal{D}_{J}$. It is known that $\theta$ is a map of Hopf algebras [5, 15] and thus it follows that $\mathcal{P}$ is a Hopf subalgebra of $\mathcal{N}$ Sym.

It is known that $\Omega$ is self dual and the dual of $\hat{\theta}$ is the inclusion map $\Omega \hookrightarrow S y m$. Hence the diagram of figure 10 dualizes producing the diagram of figure 11 . Thus the image of $\mathcal{P}$ under the abelianization map is $\Omega$. We note here that in Stembridge's work there is no consideration to the inner multiplication (namely the algebra structure on each $\mathcal{P}_{n}$ ) which is the main object of this work.

\section{Acknowledgments}

I wish to thank Louis Billera for suggesting the possible existence of the peak algebra and Richard Stanley for suggesting that exercise 7.95 [13] might be relevant to the proof. I would also like to thank Marcelo Aguiar for helpful conversations regarding the connections between the peak algebra $\mathcal{P}$ and Stembridge's peak algebra $\Pi$, and Stephanie van Willigenburg for helpful comments on this document.

\section{References}

1. D. Bayer and P. Diaconis, "Trailing the dovetail shuffle to its lair," Ann. Appl. Probab. 2(2) (1992), $294-313$.

2. F. Bergeron and N. Bergeron, "Orthogonal idempotents in the descent algebra of $B_{n}$ and applications," J. Pure Appl. Algebra 79(2) (1992), 109-129.

3. F. Bergeron, N. Bergeron, R.B. Howlett, and D.E. Taylor, "A decomposition of the descent algebra of a finite Coxeter group," J. Algebraic Combinatorics 1 (1992), 23-44.

4. F. Bergeron, A. Garcia, and C. Reutenauer, "Homomorphisms between Solomon's descent algebra," J. Algebra 150 (1992), 503-519.

5. N. Bergeron, S. Mykytiuk, F. Sottile, and S. van Willigenburg, "Shifted quasisymmetric functions and the Hopf algebra of peak functions," Discrete Math. 246 (2002), 57-66.

6. P. Cellini, "A general commutative descent algebra," J. Algebra 175 (1995), 990-1014.

7. P. Doyle and D. Rockmore, "Riffles, ruffles, and the turning algebra," preprint.

8. A.M. Garsia and C. Reutenauer, “A decomposition of Solomon's descent algebra," Adv. in Math. 77 (1989), $189-262$. 
9. I.M. Gessel, "Multipartite P-partitions and inner products of skew Schur functions," Contemp. Math. 34 (1984), 289-301.

10. J.L. Loday, Opérations sur l'homologie cyclique des algèbre commutatives, Invent. Math. 96 (1989), 205-230

11. C. Malvenuto and C. Reutenauer, "Duality between quasisymmetric functions and the Solomon descent algebra," J. Algebra 177 (1995), 967-982.

12. L. Solomon, "A Mackey formula in the group ring of a Coxeter group," J. Algebra 41(2) (1976), 255-268.

13. R.P. Stanley, Enumerative Combinatorics, Cambridge University Press, Cambridge, 1999, Vol. 2.

14. R.P. Stanley, "Ordered structures and partitions," Mem. Amer. Math. Soc. 119 (1972).

15. J.R. Stembridge, "Enriched P-partitions," Trans. Amer. Math. Soc. 349 (1997), 763-788. 